\title{
Potential and challenges of FWI with OBN data to image the Pre-salt of the Santos Basin
}

\author{
Florian Jouno, Adriano Martinez, Denis Ferreira, Daniela Donno, Adel Khalil, CGG
}

Copyright 2019, SBGf - Sociedade Brasileira de Geofísica

This paper was prepared for presentation during the $16^{\text {th }}$ International Congress of the Brazilian Geophysical Society held in Rio de Janeiro, Brazil, 19-22 August 2019.

Contents of this paper were reviewed by the Technical Committee of the $16^{\text {th }}$ International Congress of the Brazilian Geophysical Society and do not necessarily represent any position of the SBGf, its officers or members. Electronic reproduction or storage of any part of this paper for commercial purposes without the written consent of the Brazilian Geophysical Society is prohibited.

\section{Abstract}

The Santos Basin, offshore Brazil, has become one of the most prospective oil provinces in the world since the first pre-salt oil discovery in 2006. Due to the complexity of geology in this region, ocean-bottom-node (OBN) acquisition has been proposed as a solution. OBN provides low frequency signal and long-offset full-azimuth illumination, which are necessary for full wave-form inversion (FWI) to realize its potential. We will show that given the right data, as provided by OBN acquisition, a reliable FWI engine and a good starting model, we are able to derive an accurate velocity model offering the necessary resolution to improve the imaging of the salt and pre-salt sections. This brings a major step forward for imaging and model building compared to the narrow azimuth streamer data (NAZ) which have been mostly used up to now. Nevertheless, some major challenges remain. FWI is extremely dependent on the starting model used, as recording reliable very-low frequencies is still difficult. Multi-parameter FWI for anisotropy update also remains a challenge, due to the cross-talk between update of velocity and anisotropy, even with OBN acquisition. Surmounting these challenges will be arduous. Better multi-parameter FWI technology together with enhanced acquisition are still necessary.

\section{Introduction}

The Santos Basin is well known for its giant pre-salt oil and gas fields. Since 2006 and the first pre-salt oil discovery, this province has become one of the most prospective regions in the world. Today, this basin provides half of Brazil's national oil production. But understanding its plays is challenging. Pre-salt reservoirs usually lie at a depth of around $5 \mathrm{~km}$, below a thick stratified salt. The pre-salt geology is usually complex with fast carbonates, slow sediments, volcanic layers and large fault systems.

The majority of the seismic data available to assess the Santos Basin have been acquired by NAZ towed streamer surveys. These can offer reasonable understanding of the regional geology but can be insufficient to capture the local complexity of the prospects. One of the main complications with NAZ acquisitions are the limited capacity to build reliable velocity models. For that reason, velocity model building strategies have relied on complex workflows

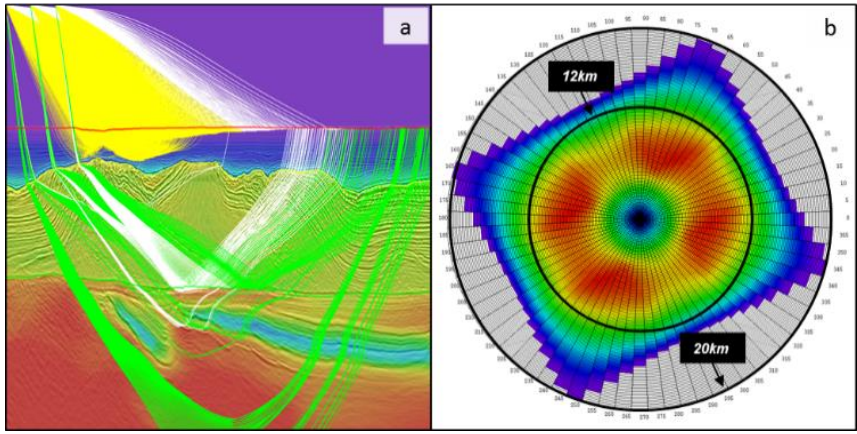

Figure 1: (a) Diving rays over the velocity model, with the water bottom (WB) horizon in red. Yellow rays (offsets from 4 to $8 \mathrm{~km}$ ) cannot penetrate deeper than top of salt. White rays (from 8 to $12 \mathrm{~km}$ ) can reach base of salt and some pre-salt layers. Green rays (from 12 to $18 \mathrm{~km}$ ) shows penetration in all pre-salt formations. (b) Rose diagrams using all nodes, with the inner and outer circumferences indicating $12 \mathrm{~km}$ and $20 \mathrm{~km}$ offset, respectively.

involving layer stripping, multiple passes of tomography, shallow diving wave-based full-waveform inversion (FWI) to update the sediments, top-of-salt (TOS) interpretation, salt-flood migration for base-of-salt (BOS) interpretation, and numerous salt scenarios. This approach is not only time consuming, but also susceptible to inaccuracies caused by human misinterpretation (Dellinger et al., 2017).

The rich low frequencies as well as the full azimuth and long offset illumination provided by OBN data open the door for more data-driven solutions using FWI. These features naturally provided by an OBN acquisition are essential for building a good velocity model using FWI (Tarantola, 1984). The long offsets allow deeper penetration of diving waves which are the main driver for FWI updates; the full-azimuth illumination can better cover complex structures such as salt and improves FWI reliability; and the rich low frequency signal alleviate inaccuracies of the starting model (Michell et al., 2017; Shen et al., 2017).

However, even with rich low frequencies, long offset, full azimuth OBN acquisition and a relatively good starting velocity model, classical FWI formulations can fail in the complex geological settings of the Santos Basin. Recent applications of Time-lag FWI (TLFWI) (Zhang et al., 2018, Wang et al., 2019) in the Gulf of Mexico have shown great potential. The Santos Basin shares many of the challenges of the Gulf of Mexico, namely complexity of salt bodies with sharp impedance contrasts and deep targets. Another feature of the Santos Basin is the salt stratification and its large velocity variations. These complexities can pose 

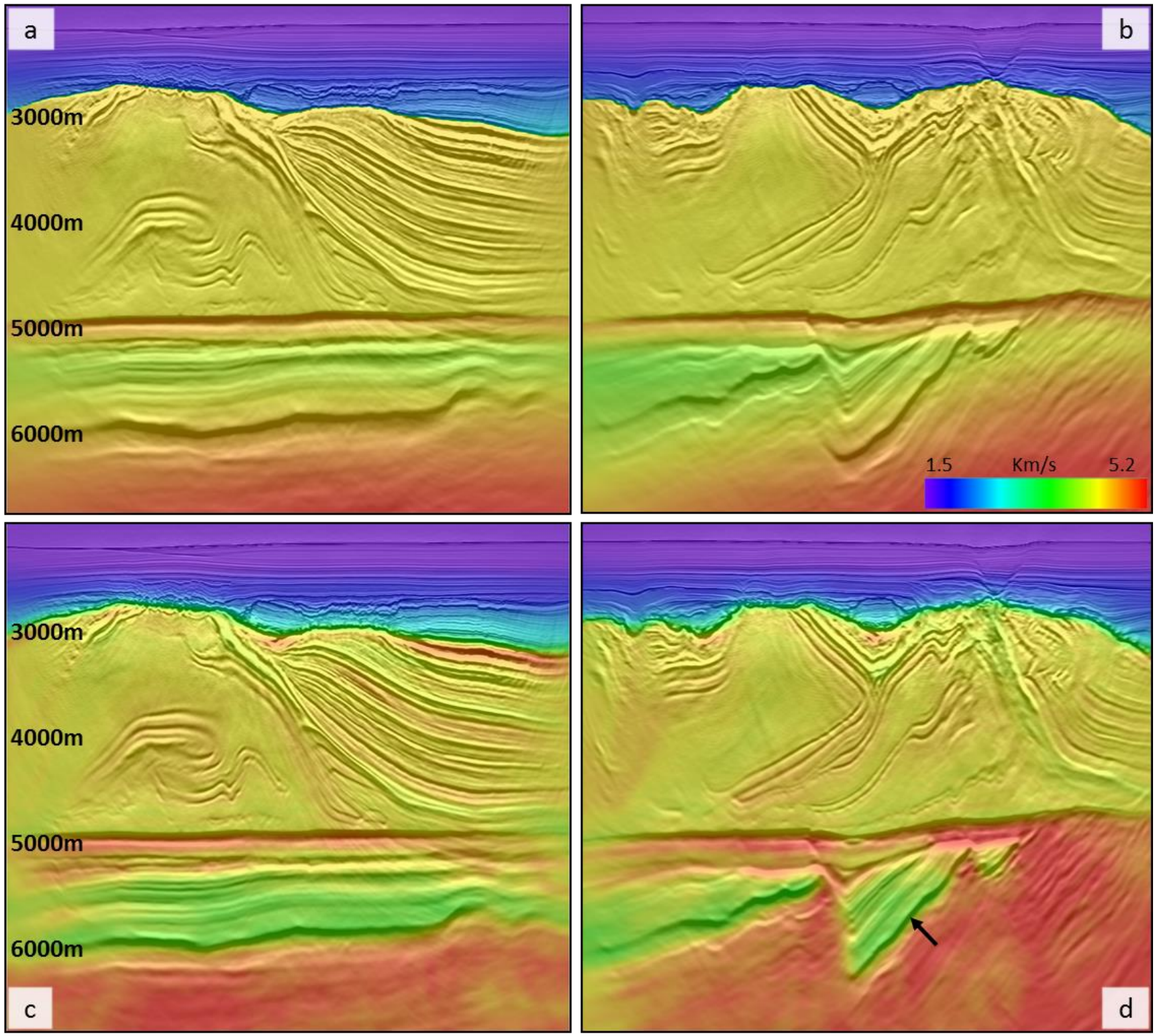

Figure 2: Inline and crossline sections with the velocity overlaid on Kirchhoff stack for (a)/(b) initial model and (c)/(d) output model from $15 \mathrm{~Hz}$ TLFWI.

additional significant challenges for classical FWI formulations. TLFWI provides a natural alternative in this geological context.

Combining OBN data, a good starting model and a robust FWI algorithm can provide valuable improvement for building velocity models in the Santos Basin and deliver a step change compared to NAZ derived results for pre-salt imaging. We explore this premise using an OBN dataset from the central part of the Santos Basin.

\section{Time-lag FWI and OBN data}

In the presence of sharp velocity contrast like salt, volcanic intrusions or carbonates, FWI can fail for two main reasons: cycle-skipping and amplitude-discrepancy between synthetic and real data. Misplaced rapid velocity transitions such as between sediments and salt can induce large timing differences between synthetic and real data beyond the half-cycle criteria, leading FWI to fall into local minima. Furthermore, the amplitude distribution of field data and synthetic data can be very different in the presence of strong reflectors like salt, which can be taken as velocity error by FWI, leading to erroneous velocity models.

To mitigate these issues and to take full advantage of the OBN benefits, we use TLFWI as proposed by Zhang et al (2018). The inversion utilizes a travel-time cost function with frequency-dependent time-windowing. The frequency dependent windowing helps to mitigate cycle skipping while the time-lag based objective function focuses mostly on the kinematics of the data, thus reducing the sensitivity to amplitude discrepancy. This, together with a weighting function based on the cross-correlation quality between 

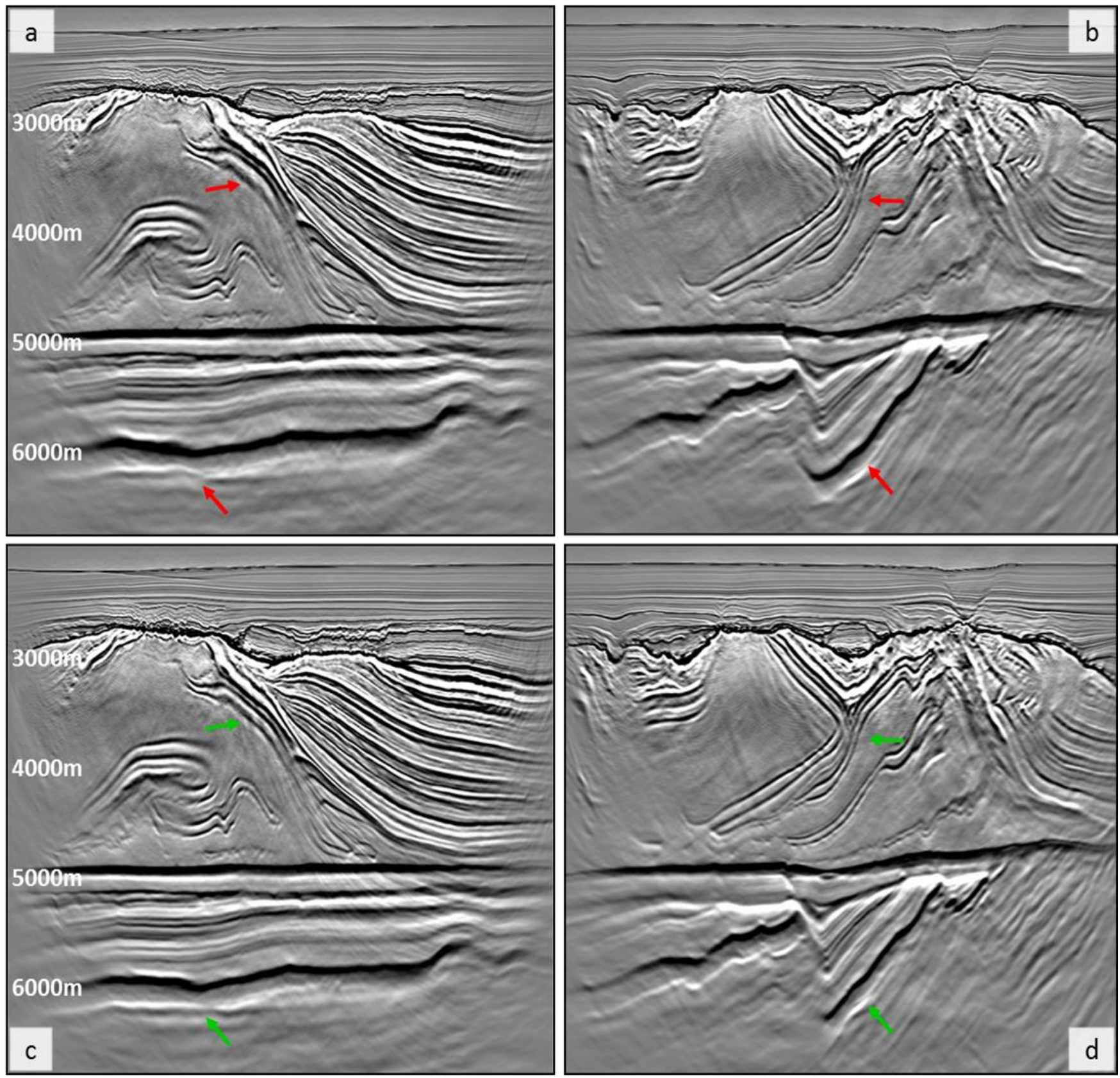

Figure 3: Inline and crossline Kirchhoff stack sections for (a)/(b) initial model and (c)/(d) output model from 15Hz Time-lag FWI. The arrows point to improvements of imaging after FWI; intra-salt events are more focus and continuous, pre-salt layers are more geologically consistent.

recorded and synthetic data promotes the robustness of the algorithm. These attributes make TLFWI a suitable candidate for Santos Basin geology, particularly for the velocity update of the salt and pre-salt regimes.

The dataset we are using is located in the central part of the Santos Basin, south of Rio de Janeiro, in water depths of about $2 \mathrm{~km}$. A fast post salt Albian layer and thick stratified salt layer covering most of the region characterize the local geology. The pre-salt regime is complex with fast carbonates, slow sediments, volcanic layers and a large fault system.

The OBN acquisition has approximately 1000 nodes covering the area, on an approximately $400 \mathrm{~m}$ by $400 \mathrm{~m}$ separation grid. The shot spacing is $50 \mathrm{~m}$ by $50 \mathrm{~m}$. Thanks to the $\sim 16 \mathrm{~km}$ maximum offset OBN data (Figure 1b), diving-waves have adequate penetration within the pre-salt layer for updating the velocity using FWI (Figure 1a). 

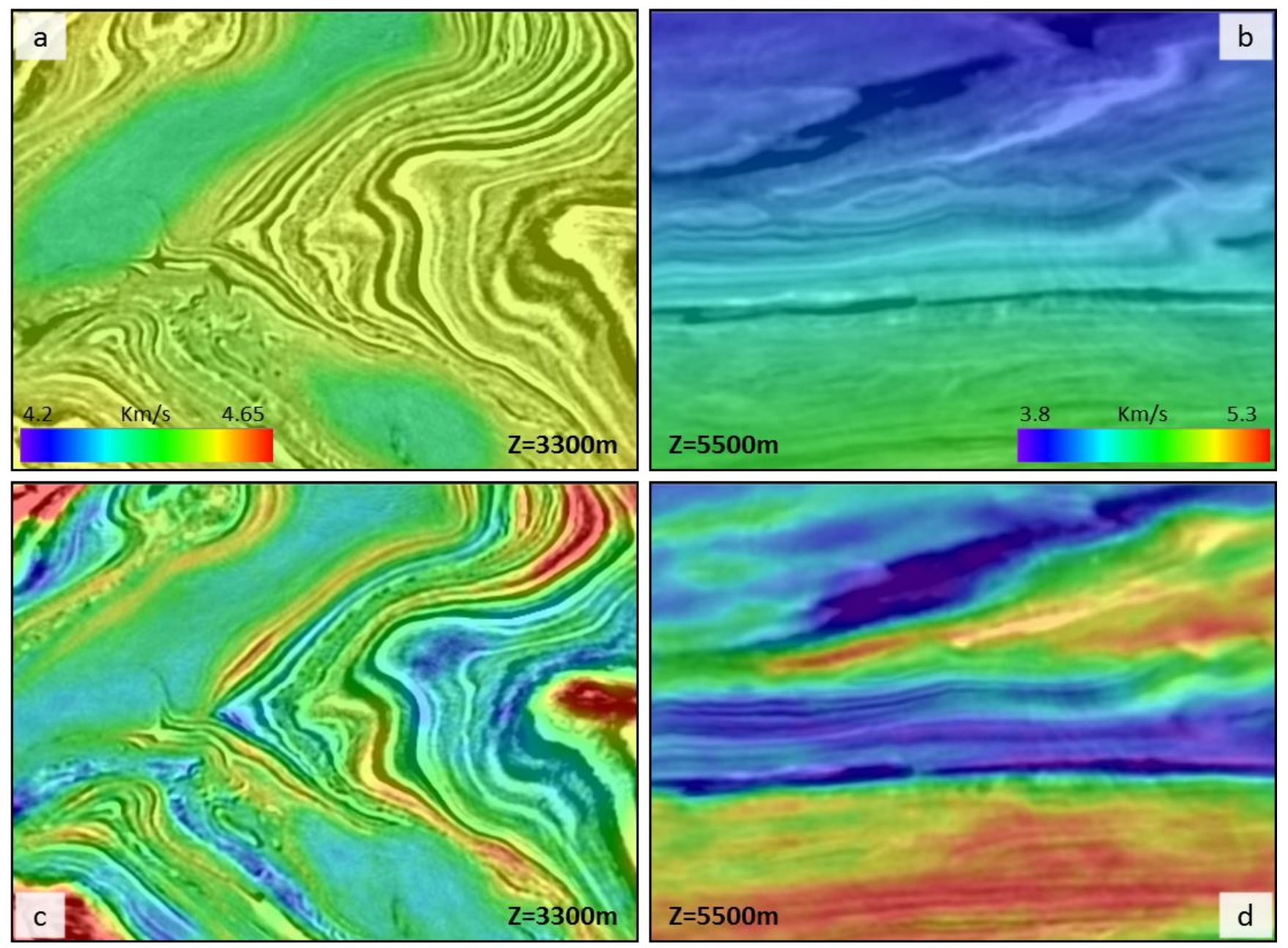

Figure 4: Depth slices, in the salt $z=3300 \mathrm{~m}$ and pre-salt $z=5300 \mathrm{~m}$, with the velocity overlaid on Kirchhoff stack for (a)/(b) initial model and (c)/(d) output model from $15 \mathrm{~Hz}$ Time-lag FWI.

\section{Exploring FWl's potential}

The input to TLFWI is the filtered and resampled raw OBN data. The initial velocity model is a smoothed version of an available legacy model built using NAZ towed streamer data. Anisotropic parameters remain unchanged compared to the legacy model. TLFWI was used to simultaneously invert for the post-salt, salt, and pre-salt sections, using frequencies from $1.6 \mathrm{~Hz}$ to $15 \mathrm{~Hz}$.

Even though the legacy velocity model provided a reasonable image, the TLFWI velocity led to a structural improvement at the pre-salt level, as shown in Figure 2. The low frequency updates $(1.6$ to $6 \mathrm{~Hz})$ offer a more geologically consistent image, correcting the regional salt velocity, enhancing the base of salt and improving fault definition in the pre-salt by capturing a slow velocity layer (black arrow in Figure 2). It is worth noting that deep presalt updates using tomography can be very challenging due to the reduced residual move-out sensitivity. The high frequency ( 6 to $15 \mathrm{~Hz}$ ) inversions offer sharper velocity contrast especially at the TOS level and provide some details in the stratified salt, the post-salt and pre-salt carbonates.

Figure 3 shows the same inline and crossline Kirchhoff stack image for the initial legacy model and after TLFWI velocity update. The more noticeable changes in imaging are in the salt and pre-salt (green arrows). Reflectors are more focused and continuous; pre-salt faults are better defined and the resolution below the base of salt is improved. Figures 4 displays depth slices, in the salt at a depth of $3300 \mathrm{~m}$ and the pre-salt at a depth of $5300 \mathrm{~m}$, with the initial legacy model, in Figure 4a-b, and the TLFWI model, in Figure 4c-d. FWI captures the intra-salt velocity variation induced by salt stratification. The depth slice in the pre-salt section (Figure 4b-d) highlights the lateral variation of velocity in the pre-salt. Figure 5 confirms the validity of the FWI model with well sonic logs at two locations. We can see that FWI gives a good low frequency representation of the velocity variations encountered in the sonic logs, in both the salt and pre-salt sections. 


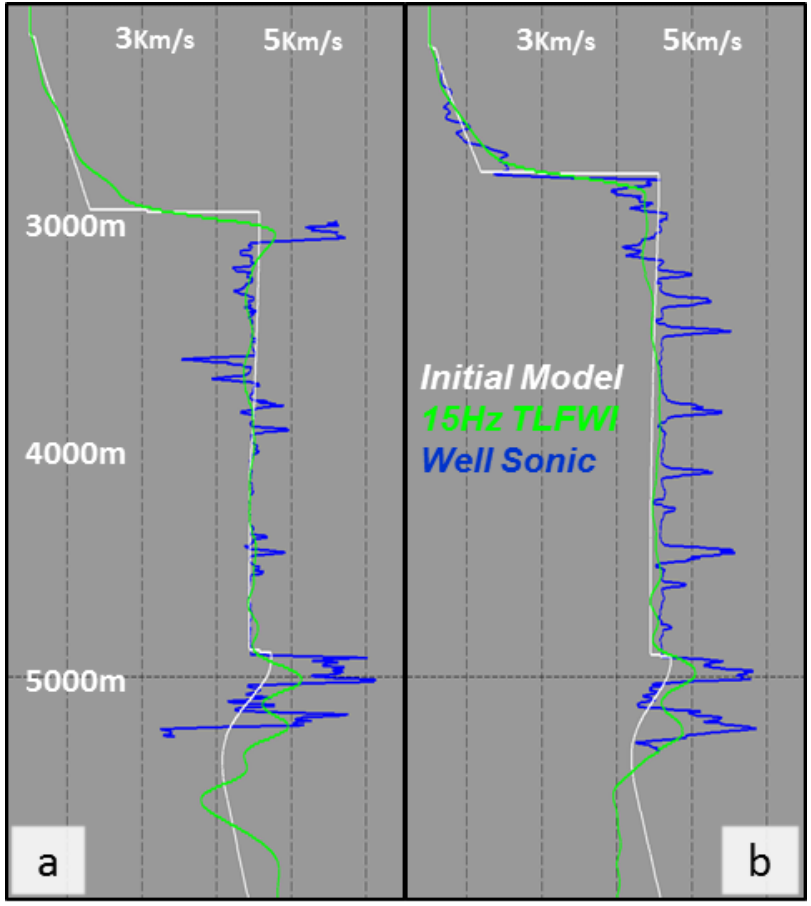

Figure 5: a) \& b) Velocity profiles at two well locations; blue profile corresponds to the sonic log, orange profile is the legacy velocity model, and green profile is the TLFWI model. After FWI, the velocity follows the sonic profile in the salt and pre-salt more accurately.

\section{Challenges and Discussions}

In the results shown here, velocity is the key focus, and anisotropy is kept unchanged throughout the inversion. However, this limits the accuracy of the updated model. Figures $7 a-b$ display the velocity and epsilon profiles at a well location for the legacy model, the FWI model, and a final model that includes a tomographic well calibration. In the pre-salt section, the use of the legacy anisotropy model limited FWI ability to converge to a more correct solution at a slow velocity layer ( 5000m). Figures $7 c-d$ display snail gathers up to $12 \mathrm{~km}$ offsets. The final model offers flatter events in the post-salt, salt and pre-salt sections.

In the current state of FWI technology, multi-parameter inversion remains challenging. Prieux et al. (2011) discuss some of the difficulties. More developments are required for FWI to be able to decouple anisotropy and velocity as well as other subsurface parameters. This is an active research field as seen in the work of Da Silva et al., (2016) and, Djebbi and Alkhalifah (2019). Joint reflection and refraction velocity inversion using extra-long offsets $(>20 \mathrm{~km})$ could theoretically facilitate the decoupling between the anisotropy and velocity terms (Allemand et al. 2017).

The OBN data used here offer rich low frequency signal compared to streamer data. Phase ring QCs (Figures 6ab) show usable signal at $\sim 1.6 \mathrm{~Hz}$ but with relatively low signal-to-noise ratio. More reliable signal is attainable at $\sim 2$ $\mathrm{Hz}$. Having this low frequency content as well as a reasonable initial model, FWI was fully data-driven with almost no human intervention. Looking further ahead at

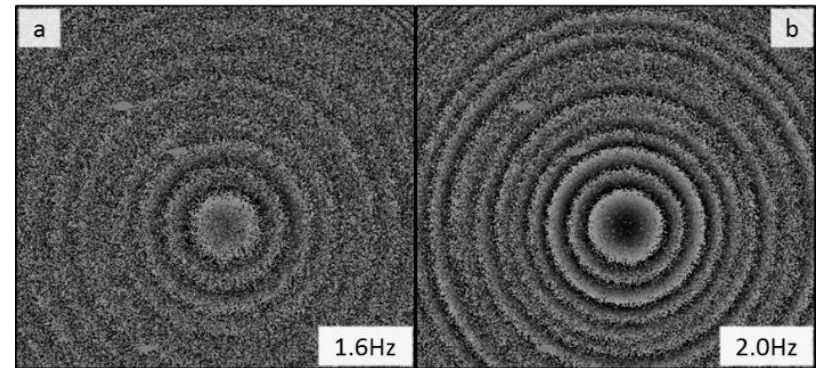

Figure 6: Phase-ring QC at (a) $1.6 \mathrm{~Hz}$ and (b) $2.0 \mathrm{~Hz}$.

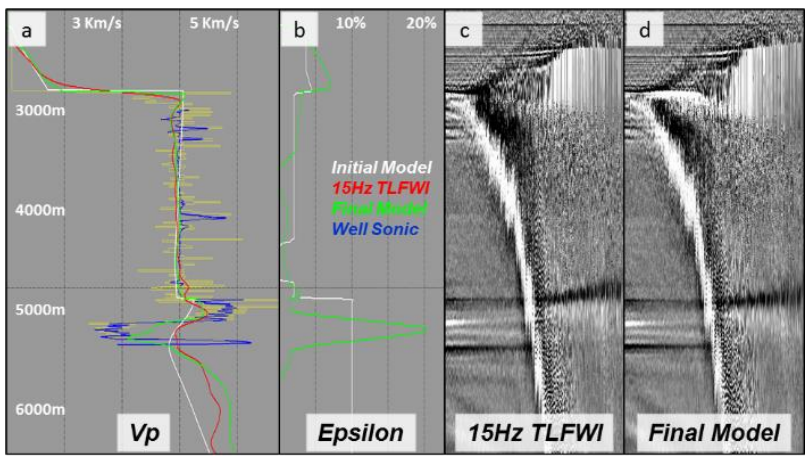

Figure 7: (a) Velocity profiles at a well location; blue profile corresponds to the sonic log, white profile to the legacy velocity model, red profile to the $15 \mathrm{~Hz}$ Time-lag FWI and green profile to the final model. (b) Epsilon profiles at a well location; white profile corresponds to the legacy Epsilon model and green profile to the final Epsilon model. (c)/(d) snail gathers up to $12 \mathrm{~km}$ offsets migrated with the $15 \mathrm{~Hz}$ FWI and the final model.

other areas where such good initial models might not be available, FWI might still require help from other technology to build the initial model, even with an equivalent $\mathrm{OBN}$ dataset. In an effort to achieve this ultimate goal of a fully automatic FWI, Dellinger et al. (2016) performed recent experiments in Gulf of Mexico with an ultra-low frequency source. The ultra-low wavenumber updates provided in this case may further reduce FWI's reliance on initial velocity models.

\section{Conclusions}

Substantial imaging improvements are achievable in complex geological settings such as in the Santos basin when combining the properties of an OBN acquisition and a reliable $\mathrm{FWI}$ formulation. Updating the anisotropy continue to be a challenge for FWI and a good starting model is necessary to overcome the low frequency limitation of the data. Improved OBN acquisitions, with ultra-low frequency and ultra-long offset, together with a reliable multi-parameter anisotropic technology will be key to obtain a more complete data driven solution.

\section{Acknowledgments}

We thank Petrobras for the discussions, technical contributions and for the permission to publish this work. We also thank CGG for permission to publish this work. 


\section{Reference}

ALLEMAND, T., A. SEDOVA, O. HERMANT, 2017, Flattening common image gathers after full-waveform inversion: the challenge of anisotropy estimation: 87th Annual International Meeting, SEG, Expanded Abstracts, 1410-1415.

DA SILVA, N. V., A. RATCLIFFE, V. VINJE, AND G. CONROY, 2016, A new parameter set for anisotropic multiparameter full-waveform inversion and application to a North Sea data set: Geophysics, 81(4), U25-U38.

DELLINGER, J., A. J. BRENDERS, J. R. SANDSCHAPER, C. REGONE, J. ETGEN, I. AHMED, AND K. J. LEE, 2017, The Garden Banks model experience: The Leading Edge, 36(2), 151-158.

DELLINGER, J., A. ROSS, D. MEAUX, A. BRENDERS, G. GESOFF, J.T. ETGEN, J. NARANJO, G. OPENSHAW, AND M. HARPER, 2016, Wolfspar®, an "FWI-friendly" ultralow-frequency marine seismic source: 86th Annual International Meeting, SEG, Expanded Abstracts, 48914885.

DJEBBI, R., T. ALKHALIFAH, 2019, Frequency domain multiparameter acoustic inversion for transversely isotropic media with a vertical axis of symmetry: Geophysics, 84(1), C1-C14.

MICHELL, S., X. SHEN, A. BRENDERS, J. DELLINGER, I. AHMED, AND K. FU, 2017, Automatic velocity model building with complex salt: Can computers finally do an interpreter's job?: 87th Annual International Meeting, SEG, Expanded Abstracts, 5250-5254.

PRIEUX, V., R. BROSSIER, Y. GHOLAMI, S. OPERTO, J. VIRIEUX, O. I. BARKVED, AND J. H. KOMMEDAL, 2011, On the footprint of anisotropy on isotropic full waveform inversion: The Valhall case study: Geophysical Journal International, 187, 1495-1515.

SHEN, X., I. AHMED, A. BRENDERS, J. DELLINGER, J. ETGEN, AND S. MICHELL, 2017, Salt model building at Atlantis with Full Waveform Inversion: 87th Annual International Meeting, SEG, Expanded Abstracts, 15071511.

TARANTOLA, A., 1984, Inversion of seismic reflection data in the acoustic approximation: Geophysics, 49(8), 12591266.

WANG, P., Z. ZHANG, J. MEI, F. LIN AND R. HUANG, 2019, Full-waveform inversion for salt: A coming of age: The Leading Edge, 38(3), 204-213.

ZHANG, Z., J. MEI, F. LIN, R. HUANG, AND P. WANG, 2018, Correcting for salt misinterpretation with fullwaveform inversion: 88th Annual International Meeting, SEG, Expanded Abstracts, 1143-1147. 